\title{
BMJ Open Longitudinal study of symptom burden in outpatients with advanced cancers based on electronic Patient-Reported Outcome (ePRO) platform: a single institution, prospective study protocol
}

To cite: Tang L, Pang Y, He Y, et al. Longitudinal study of symptom burden in outpatients with advanced cancers based on electronic Patient-Reported Outcome (ePRO) platform: a single institution, prospective study protocol. BMJ Open 2020;10:e038223. doi:10.1136/ bmjopen-2020-038223

- Prepublication history for this paper is available online. To view these files, please visit the journal online (http://dx.doi. org/10.1136/bmjopen-2020038223).

\section{LT and YP contributed equally.}

Received 04 March 2020 Revised 18 September 2020 Accepted 01 October 2020

\section{A) Check for updates}

(C) Author(s) (or their employer(s)) 2020. Re-use permitted under CC BY-NC. No commercial re-use. See rights and permissions. Published by BMJ.

${ }^{1}$ Department of PsychoOncology, Peking University Cancer Hospital \& Institute, Key laboratory of Carcinogenesis and Translational Research (Ministry of Education), Beijing, China

${ }^{2}$ School of Public Health and Management, Chongqing Medical University, Chongqing, Sichuan, China

Correspondence to

Dr Lili Tang;

tanglili_cpos@126.com

\section{ABSTRACT}

Introduction An electronic Patient-Reported Outcome (ePRO) platform is needed for implementing evidencebased symptom management in outpatients with advanced cancer. We describe the overall protocol and the methodology for measuring symptom burden, to provide critical parameters needed to implement symptom management on the ePRO platform.

Methods and analysis The study focusses on patients with advanced lung cancer, stomach cancer, oesophagus cancer, liver cancer, colorectal cancer or breast cancer. The primary outcome is the change of symptom burden. MD Anderson Symptom Inventory, and other PRO instruments (Insomnia Severity Index, Hospital Anxiety and Depression Scale, 9-item Patient Health Questionnaire and EuroQol-5 dimensions -5 levels version) were used. The secondary outcomes include feasibility of using ePRO, symptomrelated quality of life, reasons for no improvement of symptoms, defining frequency of PRO assessments and cut-points, items for screening and management of comorbidity and satisfaction with ePRO platform in patients and health providers. After initial outpatient visit for baseline assessment, ePRO system will automatically send follow-up notification seven times over 4 weeks to patients. The characteristics and changing trajectory of symptoms of patients will be described. Parameters for using PROs, such as optimal time points for follow-up and cut-off point for alert will be determined. The feasibility of ePRO platform to track the changes of target symptoms in outpatients will be evaluated.

Ethics and dissemination The study protocol and related documents were approved by the Institutional Research Board (IRB) of Peking University Cancer Hospital on 13 February 2019 (2019YJZ07). The results of this study will be disseminated through academic workshops, peerreviewed publications and conferences.

Trial registration number ChiCTR1900023560.

\section{INTRODUCTION}

According to the official report by the National Central Cancer Registry of China,
Strengths and limitations of this study

The study is a prospective cohort study with a total of seven follow-ups conducted within 4 weeks. It focusses on establishing a symptom management platform for outpatient ePRO (electronic PatientReported Outcome) and obtain the dynamic symptoms trajectory of patients outside the hospital.

- Instead of a single tumour site, the six most common tumour sites are represented: lung cancer, gastric cancer, oesophageal cancer, liver cancer, colorectal cancer and breast cancer.

- PRO data are collected using an e-questionnaire based on WeChat, a major communication application in China.

- A group of questionnaires are used to measure the comprehensive well-being of patients, mainly including MD Anderson Symptom Inventory, Insomnia Severity Index, Hospital Anxiety and Depression Scale, 9-item Patient Health Questionnaire and EuroQol-5 dimensions- 5 levels version.

cancer is the leading cause of death in China; there were 4.3 million newly diagnosed cancer cases and 2.8 million cancer deaths in 2015 in China. Lung cancer, gastric cancer, liver cancer, oesophageal cancer and colorectal cancer rank as the top five cancers in men, and breast cancer, lung cancer, gastric cancer, colorectal cancer and oesophageal cancer rank as the top five cancers in women. ${ }^{1}$ Patients with cancer suffer from various symptoms due to the disease itself and treatment-related adverse reactions. ${ }^{2-4}$ Studies have shown that one-third of the patients undergoing cancer treatments reported three or more moderateto-severe symptoms. ${ }^{5}$ The symptom burden of patients with advanced cancer is even more serious. ${ }^{45}$ If the symptoms are not addressed 
properly in time, the quality of life and daily functions will be affected negatively and even shorten the survival period of the patients.

In recent years, several studies have shown that good symptom management based on patient-reported outcome (PRO) can benefit patients with advanced cancer. For example, a single-centred study published in JAMA in 2017 showed that systematic symptom monitoring can significantly prolong the survival of patients by 5.2 months, ${ }^{6}$ which even achieved better results than most newly approved anti-cancer drugs in 2016. In 2019, a multi-centred study of web-based follow-up of PRO symptoms demonstrated once again that this approach could bring survival benefits to patients. ${ }^{7}$

However, there are few studies on effective symptom management of the patients with cancer in routine patient care in China. The clinical doctors and nurses need a help for how to quickly capture the patient's dynamic change on symptom burden in patient's routine clinical appointment under the busy work flow, and the patients also need an approach for reporting symptoms actively. A lot of the symptom management are dealt with in the outpatient clinic, however, there is no monitoring system on patients' status when they are out of the hospital.

In order to resolve this problem, we have established an electronic Patient-Reported Outcome (ePRO) platform to monitor the symptom changes when the patients have left the clinic and gone back home. The platform is designed to monitor the changes of the symptom burden through PROs among outpatients. With lack of longitudinal data of physical, psychological, social symptom burden for Chinese oncology patients, the proposed study aimed to include PROs of multiple symptom assessment tool, functioning measure and health-related quality of life measure. Since there are many differences between the Chinese culture and Western culture, the ePRO platform is also in different format. In Western countries, some of the electronic applications are through email, but email is not widely used in Chinese population especially by the elderly. We inserted the ePRO platform in the WeChat applet in mobile, for WeChat is the most popular social networking application in China. According to the latest report, there are 1.15 billion monthly active users of WeChat, over $80 \%$ population in China $;^{8}$ and according to our experiences, most of our patients, even the elderly can use WeChat.

\section{METHODS AND ANALYSIS}

\section{Study design}

This is a real-world, ongoing, longitudinal singlecentre prospective study with a total of seven follow-ups conducted within 4 weeks after the first visit of the symptom management clinic (on Day 1, Day 3, Day 7, Day 10, Day 14, Day 21 and Day 28). The flow chart of this study is shown in figure 1. After the last follow-up, a semi-structured interview will be conducted with patients

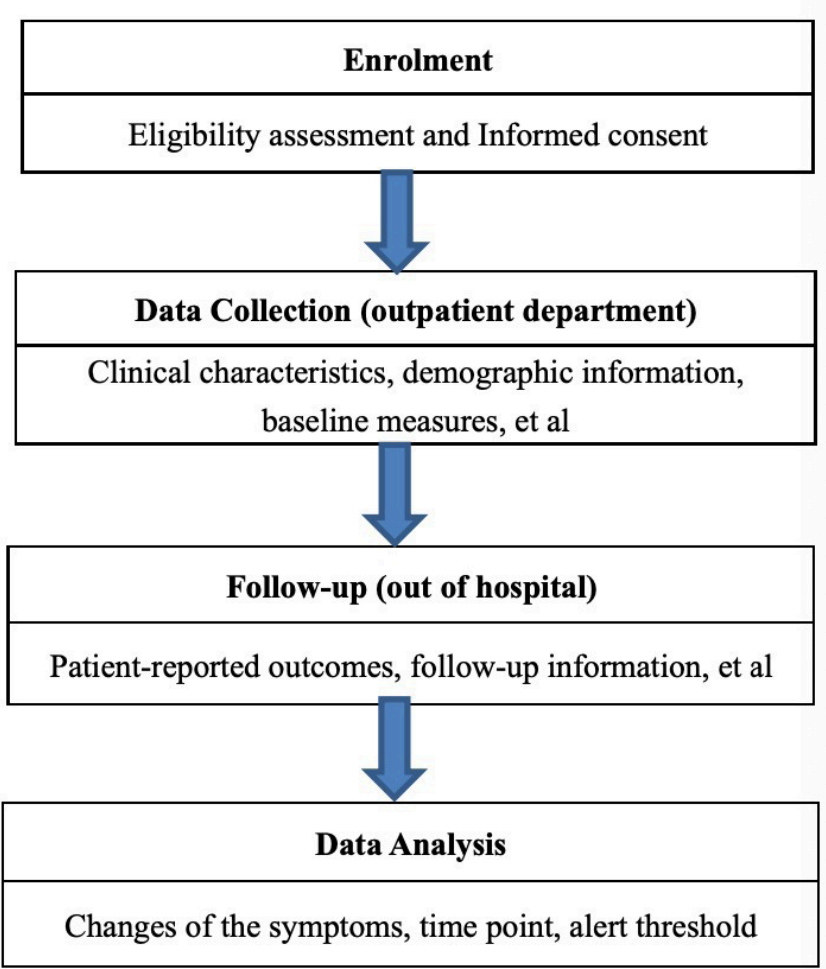

Figure 1 The flow chart of this study.

whose symptom scores worsen or not improved after 4 weeks.

\section{Setting}

This study was initiated by Department of PsychoOncology In Peking University Cancer Hospital and the study was started on $1^{\text {st }}$ June 2019 and was planned to be finished by $31^{\text {st }}$ December 2020. However, the completion data may be delayed for a few months because of the COVID-19. The site is the symptom management outpatient clinic in Peking University Cancer Hospital.

\section{Study population and eligibility criteria and recruitment}

Patients who visit the symptom management clinic for managing difficult pain or psychological symptoms. Most of the patients are referred by their oncologist, some patients were referred by other patients who had been treated in this clinic, while some patients saw advertisements. Those patients include both patients undergoing active treatment for cancer and patients receiving palliative symptomatic management. Eligible patients are required to be (1) over 18 years of age; (2) fluent in Chinese; (3) a confirmed diagnosis of advanced lung cancer, liver cancer, gastric cancer, oesophageal cancer, colorectal cancer and breast cancer; and (4) ability to give consent. Exclusion criteria include (1) a history of major severe mental disorders, unable to cooperate with the investigator; (2) poor physical or mental condition, judged by the attending physician, not able to complete the whole study; and (3) being not able to use ePRO platform. 
All eligible patients who visit the symptom management clinic at the first time will be invited to participate in this study by the doctors in this clinic. If the patients are interested in this study, a research assistant will inform patients in detail and get their written informed consent.

\section{Study objectives and outcomes}

The primary objective is systemic monitoring changes of the intensity of target symptoms (including pain, fatigue, insomnia, anxiety, depression, nausea and vomiting) in patients with advanced cancer in the symptom management clinic of Peking University Cancer Hospital during a systematic follow-up within 4 weeks.

The second objectives are as follow:

1. Evaluating the feasibility of tracking changes in target symptoms in outpatients through the ePRO follow-up system.

1. At least $50 \%$ of the patients completed self-report within 24 hours after being sent the follow-up message; more than $70 \%$ of the patients completed self-report within 24 hours after being called; and at least $70 \%$ of the patients completed all self-reports within 4 weeks.

2. The percentage of patients who met the eligibility criteria agreed to participate in the study is $80 \%$.

2. Observing the changes of quality of life in patients seeking symptom management in the Department of Psycho-Oncology within 4 weeks after initial visiting.

3 . Finding out the reasons why the symptoms of outpatients cannot be improved within 4 weeks after the first visiting through a qualitative research (interview).

4. Determining the appropriate frequency of PRO symptom screening through the description and analysis of the changes in patients' symptoms during the 4-week follow-up after initial visiting.

5. Exploring the most appropriate PRO items for the targeted symptoms of the screening.

6. Determining the alert score of each PRO symptom through the analysis of the symptom changes during the follow-up 4 weeks after initial visiting.

7. Analysing the effects of comorbidity and chronic diseases on symptom burden and symptom management of patients with cancer.

8. To explore the satisfaction with ePRO platform in patients and health provider by a focus group interview.

\section{Withdrawal criteria}

Patients undergo a brief interview with a research assistant to identify the following exclusion criteria: (1) major communication difficulties; (2) inability to commit to the required seven follow-up PRO (ie, too ill to participate); (3) cognitive impairment; (4) those who do not follow the study protocol (deliberately providing incorrect PRO data); (5) those who ask to withdraw from the research; or (6) other conditions that require withdrawal as assessed by the investigator

Study instruments, Data collection, management and monitoring, qualitative interview
Totally nine study instruments are used in this study, including six PRO instruments. The patients need to fill all the questionnaires only for three times at the baseline assessment, Day 14 and Day 28 follow-up. At other follow-ups, patients only need to fill MD Anderson Symptom Inventory with only 19 items.

\section{PRO instruments}

\section{Anderson Symptom Inventory}

MD Anderson Symptom Inventory (MDASI) ${ }^{9} 10$ is a widely used symptom inventory with 19 items (13 items for symptom severity and 6 items for interference), $0=$ nothing, $10=$ most severity. Psychometric study has shown that the Chinese version of MDASI has good reliability and validity, so that the Chinese MDASI can be used to measure the severity of multiple symptoms and their impact on function in Chinese cancer patients. Moreover, we have added five more items for specific cancer sites in our study to capture special characteristics: hot flash and upper limb lymphoedema is specific for breast cancer; cough is specific for lung cancer and swallowing difficulty is specific for oesophagus cancer; constipation is added to all cancers, because pain is one of the most common symptoms in advanced cancer patients in all types of cancer and the proportion of these patients using opioid is high. Constipation is one of the most common side effects using opioid, so we added constipation to all cancers. Additionally a single 'quality of life' item was used to anchor this study.

\section{Insomnia Severity Index}

Insomnia Severity Index (ISI) is a validated scale for measuring insomnia severity in the last 2 weeks. There are total seven items, 0 to 4 score for each item, with the sum score of 0 to 28; and 0 to 7 score indicates no insomnia; 8 to 14 score indicates subclinical insomnia; 15 to 21 score indicates moderate insomnia and 22 to 28 score indicates severe insomnia. Simplified Chinese version of ISI has been validated by Lin $e t a{ }^{11}$

\section{Hospital Anxiety and Depression Scale}

Hospital Anxiety and Depression Scale (HADS) has 14 items with a score spectrum of 0 to 4 for each item, which is used to measure the anxiety and depression for the patients in the past week. It is used for patients with somatic symptoms in the general hospitals with good reliability and validity and recommended for patients with advanced cancer or receiving palliative care. ${ }^{12}$

\section{9-Item Patient Health Questionnaire}

9-Item Patient Health Questionnaire (PHQ-9) is used to evaluate the depression of patients in the past 2 weeks. The score spectrum of symptoms severity is from $0=$ none at all to $3=$ almost every day, and the total score was from 0 to 27. Depression can be considered when the sum score is $\geq 10$. Simplified Chinese version of PHQ-9 has a good validation. ${ }^{13}$ 


\section{EuroQol-5 Dimensions- 5 Levels version}

EuroQol-5Dimensions-5 Levels (EQ-5D-5L) is a multidimensional measurement for health-related quality of life, which contains these five domains to describe patients' health: (1) mobility; (2) self-care; (3) usual activities; (4) pain/discomfort; and (5) anxiety/depression, with a scale from $0=$ no difficulty to $4=$ extremely difficulty. ${ }^{14}$

\section{Distress Thermometer}

Distress Thermometer (DT) is recommended by National Comprehensive Cancer Network in the distress management guideline and was introduced to China in 2007. DT has only one item with a scale from $0=$ no distress to $10=$ extreme distress. Problem list including five domains: practical problem, communication problem, emotion problem, physical problem and spirit and religion problem. It is recognised as the briefest tool for distress screening, especially in busy oncology clinical practice. ${ }^{16}$

The target symptoms are measured by PRO instruments: pain and fatigue are measured by MDASI; depression is measured by HADS-D and PHQ-9; anxiety is measured by HADS-A; insomnia is measured by ISI and MDASI; nausea and vomiting is measured by MDASI.

\section{General demographic and disease data questionnaire}

General demographic data includes age, sex, occupation and so on. The latter includes disease diagnosis, staging, treatment, medication and so on.

\section{ECOG (Eastern Cooperative Oncology Group score}

This is a health tool that evaluates cancer patient functional status and clinically stratifies these patients' ability to tolerate therapies, which runs from 0 to 5 , with 0 denoting perfect health and 5 death. ${ }^{17}$ This measure is done by doctors.

\section{Charlson Comorbidity Index}

Comorbid conditions are best evaluated with use of the Charlson Comorbidity Index. Many studies have shown that the impact of comorbidities is significant on the survival outcomes and prognosis of cancer patients. Here are the conditions used in the comorbidity and the number of points they are awarded: score of 1 for myocardial infarction, congestive heart failure, peripheral vascular disease, cerebrovascular disease, dementia, chronic obstructive pulmonary disease, connective tissue disease, peptic ulcer disease and diabetes mellitus (in terms of diabetes mellitus, score of 1 for uncomplicated and 2 for end organ damage); score of 2 for moderateto-severe chronic kidney disease, hemiplegia, leukaemia, malignant lymphoma, solid tumour (2 in case of presence and 6 in case tumour is metastatic), liver disease (mild 1 point and moderate-to-severe 3 points) and AIDS. ${ }^{18}$ It is determined by chart review.

\section{Data collection}

PRO data are collected using an ePRO platform. ePROhub is dedicated on ePRO clinical research on patient's data collection, assessment and management

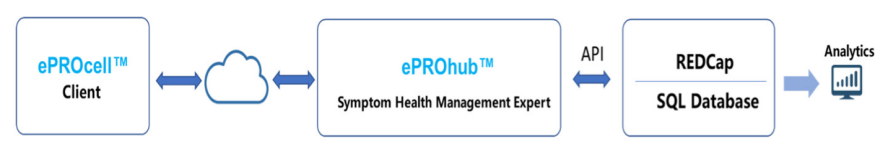

Figure 2 Peking University Cancer Hospital project case. API, application programming interface; REDCap, Research Electronic Data Capture.

launched by ePRO Vision (Beijing) Health Technology Co Ltd in 2018. The platform consist of ePROcell and ePROhub two parts. Smartphone-based client, ePROcell, is for patient's ePRO reporting and clinician's ePRO data management, intervention and project management. ePROhub platform, the EDC (electronic data capture) complied, is the cloud based symptom, management platform to store and assess patient's data, support clinicians manage patient's data and intervention activities with AI (artificial intelligence) capability and output data to other database platform for further data processing. The system could recognise the individual scores of MDASI items due to the cut-point that we set-up. For those scale that needed to be calculated for results, such as PHQ-9, were captured by WeChat first, saved in REDCap (Research Electronic Data Capture) and calculated later. Figure 2 shown below gives total picture in Beijing Cancer Hospital project case. ePROcell is fully integrated with Internet-based WeChat, the most popular and the biggest social network application in China, to maximise WeChat's advantages of world-class user experience and massive population coverage into ePRO practice enable ePRO data collection and management happening anywhere and anytime is possible. The pre-registered patients can easily click the landscape-screen to answer relevant questionnaires and send back to clinicians via cloud-based ePROhub. Figure 3 shows a screen shot to show how the platform looks to patients. The e-informed consent and e-signature is implemented before and after reporting to secure ePRO data effectiveness and integrity. The pre-defined threshold of server symptom plus triage process support the clinician to efficiently communicate with patients via ePROcell when e-alert and e-reminder occurred.

According to the pre-defined schedule, ePROcell automatically send out reminder message to patients during the reporting day, and confirmation message after patient submit. With PRO-calendar and PRO management from ePROcell, clinicians could easily review and manage project progress anytime to secure data quality, reporting progress and project quality. The ePROcell frequently visit patient's and clinical data stored in ePROhub and integrated backend database platform to support clinicians' regular patient's symptom following up and symptom management activities, which is the most efficient way to deliver effective symptom intervention and routine care with desired data-driven patient-centred care.

The cloud-based ePROhub, located in hospital's data center, is the data management platform and has dedicated data communication channel with the backend database platform REDCap, the US free of charge SQL 


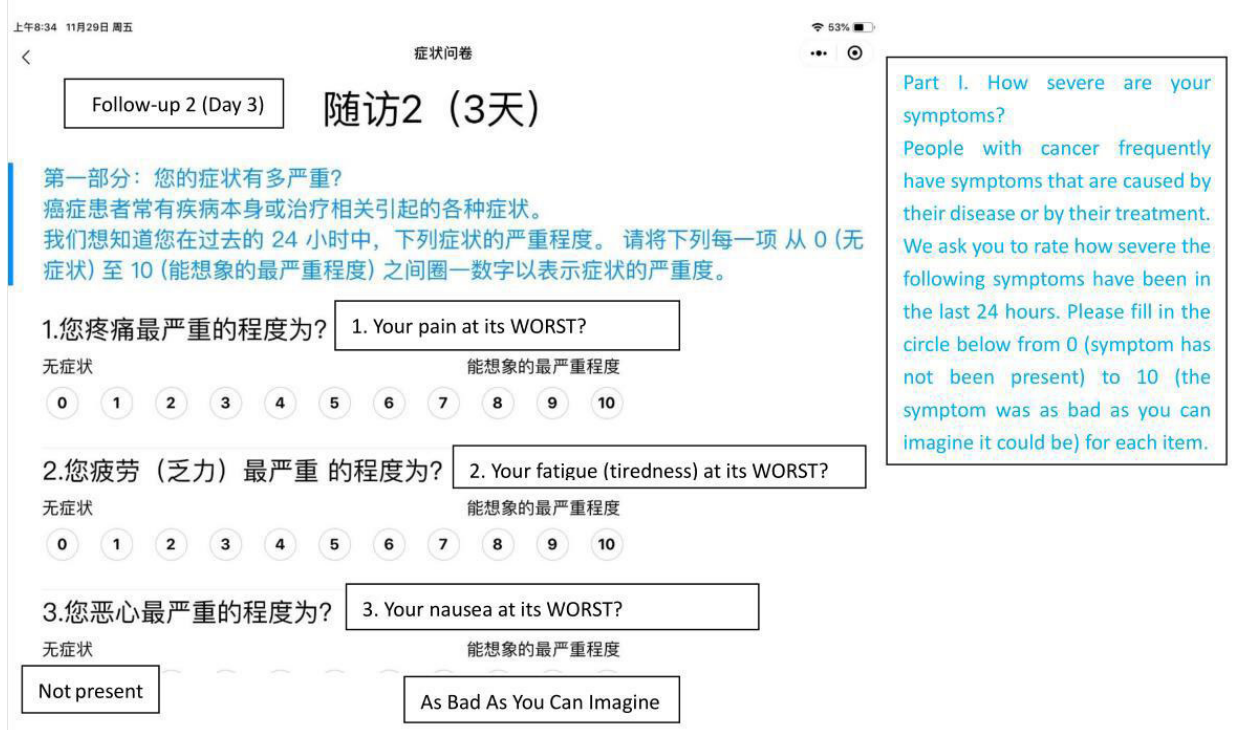

Figure 3 The screen of platform looked to patients.

database platform, via it's API (application programming interface) to synchronise pre-defined clinical data, like CRF (case report form) and scale, and patient's ePRO data. The ePRO platform can recognise the individual scores of MDASI items due to the cut-point that we set-up. For those scale that needed to be calculated for results, such as PHQ-9 are captured by WeChat first, saved in REDCap and calculated later. The output data could be converted to professional statistics programme, like SAS or SPSS, with standard data format for their final data analysis. All data are de-identified and stored in the REDCap platform. Protocol monitoring is carried out regularly by the IRB of Peking University Cancer Hospital.

\section{Semi-structured interview}

To explore the reasons for why some symptoms could not be improved within 4 weeks after the first visiting. A semi-structured interview will be done in patients with any symptom severity item score of MDASI increased or decreased less than 2 points. The guide of interview was developed with stockholder among our mean problem: why some symptoms could not be improved within 4 weeks after the first visiting. Stockholders include physicians and nurses in symptom management clinic, patients and caregivers. A face-to-face interview, will be conducted following an interview outline but if it is difficult for patients to come to the hospital, the interview will be done over the phone.

The re-coded qualitative materials will be transcribed into words and be analysed using a thematic analysis to develop a framework of topics on causes of uncontrolled symptoms. All quantitative data analysis will be performed using R V.3.6.1 and Python V.3.7.4, while the transcription of qualitative interview will be coded and analysed using NVivo V.11.0.

\section{Quality control}

Investigators received standard operating procedure training before recruiting the patients. A standardised operation process manual and an operation video has been made and distributed to all the research assistants. The group training was organised for one time while individual training was carried out one-to-one. After the training, all research assistants required to pass a test of practical operation to getting start on their official work.

There will be a Question \& Answer session to solve operation problems after around 10 cases enrolled. In addition, the practical problems that are faced by the research assistants will be shared in WeChat working group at any time.

\section{Sample size calculation}

We will use the group-based trajectory model to identify subgroups with distinct trajectories of symptom development during the 4 weeks of investigation. Previous studies showed that about $30 \%$ of the patients with advanced lung cancer reported higher symptom burden than the others. ${ }^{19}$ To obtain a 95\% CI for 30\%, we will need 148 evaluable patients, which was according to different symptom prevalence for $40 \%, 32.11 \%-47.89 \%$; $30 \%$, 22.62\%-37.38\%; $20 \%$ and $13.56 \%-26.44 \%$. Considering a $20 \%$ attrition, we will recruit 185 patients.

\section{Data analysis}

For inclusion in the final analysis, at least the baseline assessment and one successful follow-up is required. The last observation carried forward imputation method will be used to impute missing data. We will conduct a sensitivity analysis to examine whether the symptom burden of the complete cases is significantly different from that of cases with missing data. 
Continuous data will be described as mean \pm SD while categorical data will be described as number and percentage. The changing trends of target symptoms intensity over time will be presented by line charts. The improvement on the intensity of the patients' target symptoms and the PRO impact of target symptoms on patient's life in 4 weeks after the initial visiting will be analysed by general linear mixed model, while the appropriate frequency of ePRO screening for different symptoms will be determined according to significant change point of ePRO score. The impact of target symptoms intensity on quality of life, the alert value of each symptom and, most appropriate PRO items for the targeted symptoms of the screening and the effects of comorbidity and chronic diseases on symptom burden and symptom management will be analysed by regression models. Differences are considered statistically significant if the two-tailed $p$ values of $<0.05$.

\section{Patient and public involvement}

Patients and the general public were not involved in the design, recruitment and implementation of the study except development of the semi-interview guide. We have no plans of informing the study participants regarding the results of this study. However, the results will be disseminated to the applicants in the form of a published article as requested.

\section{ETHICS AND DISSEMINATION}

The protocol was approved by the IRB of Peking University Cancer Hospital on 14 May 2019 (2019YJZ34) and any amendments to the research protocol will be submitted for IRB approval. The results in this study will be first reported at relevant academic conferences, workshops and then will eventually be published in peer-reviewed journals.

\section{DISCUSSION}

Patients with advanced cancer suffered from severe symptom burden and ePRO platform could be a useful tool to monitor and to manage the patients' symptoms at home and it is urgent to incorporate ePRO into patients' electronic health record. ${ }^{20}$ This is a feasibility study to investigate the use of an ePRO platform, and this data will provide guide to apply this novel platform in clinical practice of symptom management.

The patients who visit symptom management clinic at the first time usually suffer from one or more severe symptoms and will be given drug therapy or non-drug therapy or both to address their symptoms. Usually in the first few days after their first visit, the symptoms will change rapidly. So we arrange seven follow-up on Day 1 , Day 3, Day 7, Day 10, Day 14, Day 21 and Day 28 after the first visit.

The information privacy is very important. Information of completion progress would be shown on the physician sites. Each response on WeChat requires an authorised security token to be submitted, a secured network connection ensures that collected responses were only sent to the database established in Beijing Cancer Hospital. The ePRO and data transmission network were reviewed and approved by the information security engineer of Beijing Cancer Hospital.

So far this ePRO platform has not been investigated into patient records and the ePRO platform will send a notification to remind the patients with worsening symptoms to visit the symptom management clinic as soon as possible. It is urgent to incorporate ePRO into patients' electronic health record. ePRO platform could be a useful tool to monitor and manage the patients' symptoms at home.

If the results of this study show this platform is feasible to use and is accepted by the patients and staff, our next goal is to implement ePRO into clinical practice by integrating the ePRO with patient's electronic health record. We hope this platform could be helpful for clinicians to capture their patients' symptom change in-time and offer a more flexible symptom/medication management when worsening symptoms develop.

Although there are culture differences between Chinese and Western culture, the intervention that this study describes could be replicated in many countries allowing for the use of alternative mobile platforms, and the results would be widely applicable.

Contributors LT, YP, YH and QS contributed to the study design. LT, YP, YH, XH, ZL, CZ, YZ, SH, YW, YZ, LS, BW and XL performed the study. YP, YH, XH, ZL and CZ drafted the initial manuscript. LT and QS revised the draft. All authors have reviewed and approved the final manuscript.

Funding The authors have not declared a specific grant for this research from any funding agency in the public, commercial or not-for-profit sectors.

Competing interests None declared.

Patient and public involvement Patients and/or the public were not involved in the design, or conduct, or reporting, or dissemination plans of this research.

Patient consent for publication Not required.

Provenance and peer review Not commissioned; externally peer reviewed.

Open access This is an open access article distributed in accordance with the Creative Commons Attribution Non Commercial (CC BY-NC 4.0) license, which permits others to distribute, remix, adapt, build upon this work non-commercially, and license their derivative works on different terms, provided the original work is properly cited, appropriate credit is given, any changes made indicated, and the use is non-commercial. See: http://creativecommons.org/licenses/by-nc/4.0/.

\section{ORCID iD}

Lili Tang http://orcid.org/0000-0003-1524-4617

\section{REFERENCES}

1 Chen W, Zheng R, Baade PD, et al. Cancer statistics in China, 2015. CA Cancer J Clin 2016;66:115-32.

2 Reilly CM, Bruner DW, Mitchell SA, et al. A literature synthesis of symptom prevalence and severity in persons receiving active cancer treatment. Support Care Cancer 2013;21:1525-50.

3 Dantzer R, Meagher MW, Cleeland CS. Translational approaches to treatment-induced symptoms in cancer patients. Nat Rev Clin Oncol 2012;9:414-26.

4 Henry DH, Viswanathan HN, Elkin EP, et al. Symptoms and treatment burden associated with cancer treatment: results from a cross- 
sectional national survey in the U.S. Supportive Care in Cancer 2008:16:791-801.

5 Cleeland CS, Zhao F, Chang VT, et al. The symptom burden of cancer: evidence for a core set of cancer-related and treatmentrelated symptoms from the eastern cooperative Oncology Group symptom outcomes and practice patterns study. Cancer 2013;119:4333-40.

6 Basch E, Deal AM, Dueck AC, et al. Overall survival results of a trial assessing patient-reported outcomes for symptom monitoring during routine cancer treatment. JAMA 2017;318:197-8.

7 Denis F, Basch E, Septans A-L, et al. Two-Year survival comparing web-based symptom monitoring vs routine surveillance following treatment for lung cancer. JAMA 2019;321:306-7.

8 Tencent. "Tencent Announces 2019 Third Quarter Results," Tencent, 2019. Available: https://cdc-tencent-com-1258344706.image. myqcloud.com/uploads/2019/11/13/8b98062831f2f28d9cb46162 22a4d3c3.pdf

9 Cleeland CS, Mendoza TR, Wang XS, et al. Assessing symptom distress in cancer patients: the M.D. Anderson symptom inventory. Cancer 2000;89:1634-46.

10 Wang XS, Wang Y, Guo H, et al. Chinese version of the M. D. Anderson symptom inventory: validation and application of symptom measurement in cancer patients. Cancer 2004:101:1890-901.

11 Lin R-M, Xie S-S, Yan W-J, et al. Factor structure and psychometric properties of the insomnia severity index in mainland China. Soc Behav Pers 2018;46:209-18.

12 Guo Hua Z, Ming Zhi XU. Factorial structure of the hospital anxiety and depression scale in outpatients with somatic disease. Chinese Journal of Clinical Psychology 2006;14:591-2.
13 Chen S, Fang $\mathrm{Y}$, Chiu $\mathrm{H}$, et al. Validation of the nine-item patient health questionnaire to screen for major depression in a Chinese primary care population. Asia Pac Psychiatry 2013;5:61-8.

14 Luo N, Liu G, Li M, et al. Estimating an EQ-5D-5L value set for China. Value Health 2017;20:662-9.

15 Liu L, Li S, Wang M, et al. Comparison of EQ-5D-5L health state utilities using four country-specific tariffs on a breast cancer patient sample in mainland China. Patient Prefer Adherence 2017;11:1049-56.

16 Tang L-L, Zhang Y-N, Pang Y, et al. Validation and reliability of distress thermometer in Chinese cancer patients. Chin $J$ Cancer Res 2011;23:54-8.

17 Oken MM, Creech RH, Tormey DC, et al. Toxicity and response criteria of the eastern cooperative Oncology Group. Am J Clin Oncol 1982;5:649-56.

18 Charlson ME, Pompei P, Ales KL, et al. A new method of classifying prognostic comorbidity in longitudinal studies: development and validation. J Chronic Dis 1987:40:373-83.

19 Cleeland CS, Mendoza TR, Wang XS, et al. Levels of symptom burden during chemotherapy for advanced lung cancer: differences between public hospitals and a tertiary cancer center. $J$ Clin Oncol 2011;29:2859-65.

20 Marandino L, Necchi A, Aglietta M, et al. COVID-19 emergency and the need to speed up the adoption of electronic patientreported outcomes in cancer clinical practice. JCO Oncol Pract 2020;16:295-8 